\section{Bending the Poggendorff parallels and the rules of inference: A note on Brigell and Uhlarik (1980)}

\author{
PETER WENDEROTH \\ University of Sydney, Sydney 2006, Australia
}

Brigell and Uhlarik (1980) claim to have adduced evidence for the role of perceived angular distortion in the Poggendorff illusion. They may be right; but aspects of their experimental design and logic should be considered carefully before accepting the conclusions they seek to draw.

The rationale of the experiments was to vary angles $\alpha$ and $\beta$ independently (Figure 1A), rather than conjointly as has occurred in most other experiments concerned with angular effects. To achieve their aim, the authors used figures with bent "parallels" (Figure 1, B to D), and Figure 1, A to D, in essence reproduces their Figure 1.

Consider some of the results. Brigell and Uhlarik found that: (a) largest errors, in the usual Poggendorff direction, occurred when $\beta$ was $120 \mathrm{deg}$ (Figure 1B), and these were on the order of 3 to $4 \mathrm{deg}$; (b) quite small errors were obtained with the "standard" figure, in which $\alpha+\beta=180$ deg (Figure 1A); (c) virtually zero errors occurred when $\alpha=\beta$ (Figure 1C); and (d) reversed errors occurred, and were maximum, when $\alpha=45 \mathrm{deg}$ (or $30 \mathrm{deg}$ ) and $\beta=15 \mathrm{deg}$ (Figure 1D).

All of these results are explicable, in principle, in terms of variables which may have nothing to do with the basic mechanisms of the Poggendorff effect. First, the repulsion of one line by another at a neighboring orientation (the tilt illusion) is maximum for orientation differences on the order of 10-15 deg (Carpenter \& Blakemore, 1973), even in the oblique meridian (O'Toole \& Wenderoth, 1977). Thus, the oblique test lines ( $\mathrm{X}$ and $\mathrm{Y}$ in Figure 1) would be expected to be distorted in orientation in Figure 1D so that both $\mathrm{X}$ and $\mathrm{Y}$ would appear to be tilted more counterclockwise. This is so because the repulsion effect of the nonvertical "parallel" segment, $15 \mathrm{deg}$ from $X$ and $Y$, would outweigh the effect of the vertical "parallel"' segment, 30 or 45 deg away. Hence, the net tilt illusion would result in X's appearing to point above $Y$ (or $Y$ below $X$ ), the reverse "Poggendorff effect" that Brigell and Uhlarik obtained.

It is not surprising that only small errors occurred with the "standard" figure (Figure 1A) because in this version, where $X$ and $Y$ bisect the parallels, the

Preparation of this note was supported by Australian Research Grants Committee, Grant A 28815547 I.

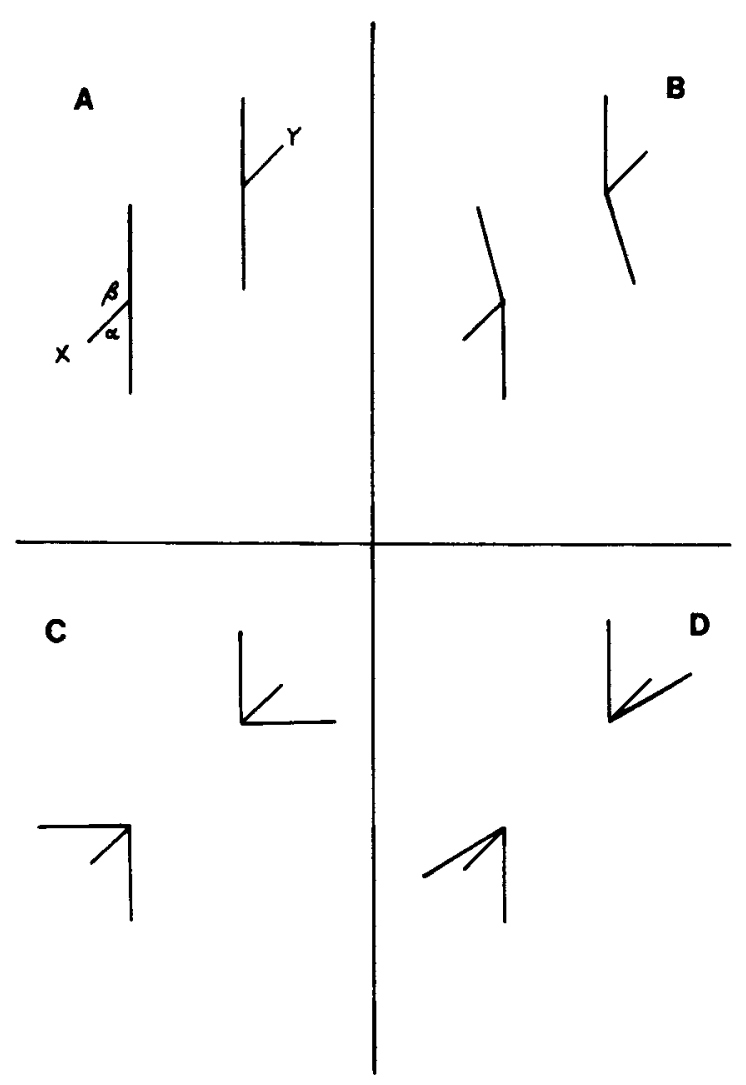

Figure 1. Stimulus figures used by Brigell and Uhlarik.

perspective cue reduces the effect to near zero, as Gillam (1971) has shown. This, of course, does not imply, necessarily, that depth cues cause the illusion in the first place; it implies merely that depth information can be used to counter the effect. This point is considered in more detail below.

Finally, while the negligible effect in Figure 1C could be due to equal-but-opposite angular effects (tilt illusions), it could also be that the symmetry of the figure overrides other factors, enabling veridical judgments to be made at some holistic level rather than at the more usual local level of processing. There is other evidence for the role of holistic (Gestalt?) factors in the Poggendorff effect (e.g., Wenderoth \& Wade, 1981).

Consequently, since angular effects might summate to reverse the illusions in $1 \mathrm{D}$, depth cues might reduce the effect in $1 \mathrm{~A}$, and symmetry might eradicate the illusion in $1 \mathrm{C}$, it is hardly surprising that the largest effects occurred in 1B. This analysis of the results gains some credence from the fact that, even then, the largest effect obtained was 3-4 deg, about half the usual, full Poggendorff effect. 
The general point is that if manipulation of a variable (P) affects the magnitude of an illusion, this does not necessarily imply that $P$, rather than some other variable $(Q)$, produces the effect in the first instance. This point has already been made in relation to depth cues (see above). A favorite example of those who wish to argue that the Poggendorff obliques bow or bend at the intersect is to draw the figure with obliques physically counterbowed at the intersect (e.g., see Glass, 1970). That this manipulation reduces the effect is hardly surprising, since the ends of the obliques physically have been moved in the direction counter to the illusion itself. To conclude from such a demonstration that straight obliques are seen bowed in the opposite direction would be a non sequitur. One could equally well eradicate the effect by raising or lowering one oblique, out of true alignment, or by increasing the separation between the parallels so that the obliques were not aligned but appeared to be. None of these manipulations necessarily bears in the slightest on the origin of the Poggendorff illusion itself.

Similarly, then, to construct figures in which angular distortions, or other effects, either counter the il- lusion or enhance it is not to reveal, necessarily, anything about the fundamental determinants of the Poggendorff effect. It would seem prudent to bear this in mind when considering the data reported by Brigell and Uhlarik (1980).

\section{REFERENCES}

Brigell, M., \& Uhlarik, J. Bending the parallels of the Poggendorff figure. Bulletin of the Psychonomic Society, 1980, 16, 1-4. Carpenter, R. H. S., \& Blakemore, C. Interactions between orientations in human vision. Experimental Brain Research, $1973,18,287-303$.

Gillam, B. A depth processing theory of the Poggendorff illusion. Perception \& Psychophysics, 1971, 10, 211-216.

Glass, L. Effect of blurring on perception of a simple geometric pattern. Nature, 1970, 228, 1341-1342.

O'Toole, B., \& Wenderoth, P. The tilt illusion: Repulsion and attraction effects in the oblique meridian. Vision Research, $1977,17,367-374$.

Wenderoth, P., \& WADE, N. An investigation of line and dot forms of the Müller-Lyer and Poggendorff illusions. Quarterly Journal of Experimental Psychology: Human Experimental Psychology, in press.

(Received for publication January 30, 1981; accepted January 30,1981 .) 